\title{
Acknowledgment of Reviewers
}

As we complete the 2017 publication cycle of Psychiatric Rehabilitation Journal, we take this opportunity to acknowledge the valued contributions of our PRJ reviewers and thank them for their efforts. With their expertise and support, we published four issues of Psychiatric Rehabilitation Journal this year. Our reviewers have graciously provided their time and expert guidance to ensure that authors receive feedback and direction to improve their submissions. Authors, in turn, express their appreciation of reviewers' input, and comment that their articles, brief reports, and Speaking Out contributions are much improved as a result. In addition to acknowledging and thanking our Editorial Review Board, we extend our sincere thanks and gratitude to the individuals listed on this page for their time, expertise, and commitment to conduct reviews for Psychiatric Rehabilitation Journal during the time period of October 2016 to October 2017.

\section{Kelly Allott}

Oyenike Balogun-Mwangi

Richard M. Balser

Deborah Becker

Dror Ben-Zeev

Crystal R. Blyler

Jane K. Burke-Miller

Veronica Carey

Cori Cather

Amy Neubauer Cohen

Francesca Coniglio

Patrick W. Corrigan

Nicole DeTore

Amy L. Drapalski
Shaun M. Eack

Vasudha Gidugu

Crystal M. Glover

Ingrid Goldstrom

Jennifer D. Gottlieb

Eric Granholm

Benjamin Henwood

Carol Irvin

Caroline Kaufmann

Sean A. Kidd

Eoin Killackey

Kathleen Anne Klik

Julie Kreyenbuhl

Matthew Kurtz
Heather Leutwyler

Rachel Loewy

Paul Lysaker

Alan G. McGuire

Colleen E. McKay

Alice Medalia

Piper Meyer-Kalos

Lisa Mueller

Jennifer Nicholas

Wendy Nilsen

Valerie A. Noel

Maria O'Connell

Laysha Ostrow

Myra Piat
Armando J. Rotondi

Paula Schnurr

Elizabeth Siantz

Victoria Stanhope

Laura Stull

Amir Tal

John Torous

Geoffrey Waghorn

Melissa SuzanneMaria Wattenberg

Philip T. Yanos 